This article may be downloaded for personal use only. Any other use requires prior permission of the author and AIP Publishing. This article appeared in Surface Science Spectra, 9(1), 260-265 and may be found at https://doi.org/10.1116/11.20020802.

\section{Alkyl Monolayers on Silica Surfaces Prepared from Neat, Heated (Tridecafluoro-1,1,2,2-tetrahydrooctyl)-1- dimethylchlorosilane Analyzed by XPS}

\author{
Ghaleb A. Husseini, Eric T. Sevy, Matthew C. Asplund, Justin Peacock, \\ and Matthew R. Linford \\ Department of Chemistry and Biochemistry, Brigham Young University, Provo, Utah 84602
}

(Received 21 August 2002; accepted 18 November 2003; published 12 March 2004)

Silane monolayers on silica, prepared from mono-, di-, and trichlorosilanes, are widely used in industry for surface functionalization and modification. However, unlike di- and trichlorosilanes, monochlorosilanes are particularly easy to work with because they can dimerize, but not polymerize, upon reaction with water. Typically, an organic solvent is used when depositing a silane monolayer. Here we show XPS spectra of monolayers of (Tridecafluoro-1,1,2,2-tetrahydrooctyl)1-dimethylchlorosilane on silicon oxide (silicon wafer) prepared using a rapid, solvent-free approach. Reaction conditions are $60{ }^{\circ} \mathrm{C}$ for $10 \mathrm{~min}$ using the neat (pure) compound, and no inert atmosphere or special treatment of the compound is required. (C) 2004 American Vacuum Society. [DOI: 10.1116/11.20020802]

Keywords: $x$-ray photoelectron spectroscopy; silane; alkylation; monochlorosilanes

\begin{tabular}{l} 
Accession \# 00767 \\
Technique: XPS \\
Host Material: Monolayer \\
(Tridecafluoro-1,1,2,2- \\
tetrahydrooctyl)-1 \\
dimethylchlorosilane/Si \\
Instrument: Surface Science \\
$\quad$ Laboratories, Inc. SSX-100 \\
Major Elements in Spectrum: C, Si, \\
O, F \\
Minor Elements in Spectrum: none \\
Printed Spectra: 5 \\
Spectra in Electronic Record: 5 \\
Spectral Category: technical \\
\hline
\end{tabular}

PACS: $82.80 . P v, 81.05 . \mathrm{Lg}$

\section{INTRODUCTION}

Silanes (Ref. 1) attach to silica surfaces by reacting with surface silanols ( $\mathrm{Si}-\mathrm{OH})$. While monolayers prepared from monochlorosilanes are more subject to hydrolysis than those derived from diand trichlorosilanes, surface functionalization with the monofunctionalized compounds is generally more straightforward because they cannot polymerize. Numerous preparations of silane monolayers on surfaces have been reported in the literature. For example, Maoz and Sagiv first showed that alkyl monolayers can be prepared on planar silicon using trichlorosilanes (Ref. 2). Recognizing the advantages of mono- over di- and trichlorosilanes, Rabolt and co-workers described a gas phase procedure for depositing perfluorinated alkyldimethylchlorosilanes on $\mathrm{SiO}_{2}$ (Ref. 3). Both Linton and co-workers (Ref. 4) and Watts and co-workers (Ref. 5) deposited monolayers of alkyldimethlychlorosilanes, including $\mathrm{ClSi}\left(\mathrm{CH}_{3}\right)_{2}\left(\mathrm{CH}_{2}\right){ }_{17} \mathrm{CH}_{3}$ 2-tetrahydrooctyl)-1-dimethylchlorosilane, onto silica particles using an organic solvent. Typical surface modifications for know procedures call for exposing a surface to a heated, dilute solution of a silane under inert atmosphere. In contrast, here we take clean, dry, native-oxide-terminated silicon $(1.5-2.0 \mathrm{~nm})$, place liquid, neat (Tridecafluoro1,1,2,2-tetrahydrooctyl)-1-dimethylchlorosilane on its surface, and heat it in an oven at $60{ }^{\circ} \mathrm{C}$ for $10 \mathrm{~min}$ (relative humidity $=70 \%$ ). After reacting, the surface is cleaned, dried, loaded into an XPS UHV chamber, and analyzed by XPS, which showed carbon levels consistent with monolayer quantities of surface alkyl chains (ellipsometric thickness is approximately $0.6-0.7 \mathrm{~nm}$, advancing contact angle $\theta_{a}=103.2^{\circ} \pm 0.5^{\circ}$, receding contact angle $\left.\left(\theta_{r}\right)=86.8^{\circ} \pm 2.5^{\circ}\right)$. The results of using this new facil surface silanization technique (for 5 different silanes) is summarized in a note published in Langmuir (Ref. 6).

\section{SPECIMEN DESCRIPTION}

Host Material: Monolayer (Tridecafluoro-1,1,2,2-tetrahydrooctyl)-1 dimethylchlorosilane/Si

CAS Registry \#: 102448-47-1
Host Material Characteristics: homogeneous; solid; single crystal; semiconductor; ceramic; glass; thin film; coating

Chemical Name: silicon/silicon oxide

Source: Montco Silicon Technologies, Inc.

Host Composition: $\mathrm{Si} / \mathrm{SiO}_{2}$

Form: single crystal wafer, $p$-type

Lot \#: W9969

Structure: Si (100)

As Received Condition: not specified

Analyzed Region: same as host material

Ex Situ Preparation/Mounting: The silicon surfaces were first cleaned with a solution of $\mathrm{NH}_{4} \mathrm{OH}$ (conc.): $\mathrm{H}_{2} \mathrm{O}_{2}$ (conc.) $(50: 50)(\mathrm{v} / \mathrm{v})$ for $30 \mathrm{~min}$ at room temperature. They were then rinsed with water and finally washed with $5 \%$ vol. $\mathrm{HCl}$ (conc.) for $1 \mathrm{~h}$. After reaction in the oven (for $10 \mathrm{~min}$ at $60{ }^{\circ} \mathrm{C}$ ), the wafers were rinsed with acetone, cleaned with a soft artists brush using a $2 \%$ sodium dodecyl sulfate solution in water, and placed in a Soxhlet apparatus overnight using $m$-xylene (b.p. $\sim 139{ }^{\circ} \mathrm{C}$ ) as the extraction solvent. The samples were then removed from the Soxhlet, rinsed with water, dried, and mounted into the XPS machine. (Note: source beam size on the instrument was not well characterized and may be up to twice as large as the manufacturer's values given here [See Source Beam Size at Specimen Surface].) Warning: this procedure should not be attempted with volatile silanes. Fumes from a volatile organic compound are potentially explosive. In addition, the $\mathrm{NH}_{4} \mathrm{OH} / \mathrm{H}_{2} \mathrm{O}_{2}$ cleaning solution is extremely caustic and should be used with great care.

In Situ Preparation: none

Charge Control: none

Temp. During Analysis: $298 \mathrm{~K}$

Pressure During Analysis: $<2.053 \times 10^{-7} \mathrm{~Pa}$ 


\section{INSTRUMENT DESCRIPTION}

Manufacturer and Model: Surface Science Laboratories, Inc. SSX-100

Analyzer Type: spherical sector

Detector: resistive anode position detector

Number of Detector Elements: 128

INSTRUMENT PARAMETERS COMMON TO ALL SPECTRA

\section{Spectrometer}

Analyzer Mode: constant pass energy

Throughput $\left(\boldsymbol{T}=\boldsymbol{E}^{N}\right): \quad N=0$

Excitation Source Window: $10 \mu \mathrm{m}$ Mylar

Excitation Source: $\mathrm{Al} K_{\alpha}$ monochromatic

Source Energy: $1486.6 \mathrm{eV}$

Source Strength: $200 \mathrm{~W}$

Source Beam Size: $0.8 \mathrm{~mm} \times 0.8 \mathrm{~mm}$

Signal Mode: multichannel direct

\section{Geometry}

Incident Angle: $55^{\circ}$

Source to Analyzer Angle: $70.8^{\circ}$
Emission Angle: $55^{\circ}$

Specimen Azimuthal Angle: $0^{\circ}$

Acceptance Angle from Analyzer Axis: $30^{\circ}$

Analyzer Angular Acceptance Width: $30^{\circ} \times 30^{\circ}$

\section{ACKNOWLEDGMENTS}

The authors acknowledge the help of Yit-Yian Lua at Brigham Young University in using the XPS.

\section{REFERENCES}

1. E. P. Plueddemann, Silane Coupling Agents (Plenum, New York, 1991).

2. R. Maoz and J. Sagiv, J. Colloid Interface Sci. 100, 465 (1984).

3. P. W. Hoffmann, M. Stelzle, and J. F. Rabolt, Langmuir 13, 1877 (1997).

4. S. J. Simko, M. L. Miller, and R. W. Linton, Anal. Chem. 57, 2448 (1985).

5. V. A. Brown, D. A. Barrett, P. N. Shaw, M. C. Davies, H.J. Ritchie, P. Ross, A. J. Paul, and J. F. Watts, Surf. Interface Anal. 21, 263 (1994).

6. G. A. Husseini, J. Peacock, A. Sathyapalan, L. W. Zilch, M. C. Asplund, E. T. Sevy, and M. R. Linford, Langmuir 19, 5169 (2003).

\begin{tabular}{lccccccc}
\hline \multicolumn{7}{c}{ SPECTRAL FEATURES TABLE } \\
\hline $\begin{array}{l}\text { Spectrum } \\
\text { ID \# }\end{array}$ & $\begin{array}{c}\text { Element/ } \\
\text { Transition }\end{array}$ & $\begin{array}{c}\text { Peak } \\
\text { Energy } \\
(\mathbf{e V})\end{array}$ & $\begin{array}{c}\text { Peak Width } \\
\text { FWHM } \\
(\mathbf{e V})\end{array}$ & $\begin{array}{c}\text { Peak Area } \\
\text { (counts) }\end{array}$ & $\begin{array}{c}\text { Sensitivity } \\
\text { Factor }\end{array}$ & $\begin{array}{c}\text { Concen- } \\
\text { tration } \\
\text { (at. \%) }\end{array}$ & $\begin{array}{c}\text { Peak } \\
\text { Assignment }\end{array}$ \\
\hline $00767-02$ & Si $2 p$ & 99.4 & 1.3 & 13783 & 0.9 & 29.9 & $\ldots$ \\
$00767-03$ & C $1 s$ & 291.9 & 2.2 & 12714 & 1.00 & 17.4 & $\ldots$ \\
$00767-04$ & O $1 s$ & 532.2 & 2.3 & 30383 & 2.49 & 28.1 & $\cdots$ \\
$00767-05$ & F $1 s$ & 688.9 & 3.2 & 49287 & 3.32 & 28.9 & $\ldots$ \\
\hline
\end{tabular}


ANALYZER CALIBRATION TABLE

\begin{tabular}{|c|c|c|c|c|c|c|c|}
\hline $\begin{array}{l}\text { Spectrum } \\
\text { ID \# }\end{array}$ & $\begin{array}{l}\text { Element/ } \\
\text { Transition }\end{array}$ & $\begin{array}{c}\text { Peak } \\
\text { Energy } \\
(\mathrm{eV})\end{array}$ & $\begin{array}{c}\text { Peak Width } \\
\text { FWHM } \\
(\mathrm{eV})\end{array}$ & $\begin{array}{c}\text { Peak Area } \\
\text { (counts) }\end{array}$ & $\begin{array}{c}\text { Sensitivity } \\
\text { Factor }\end{array}$ & $\begin{array}{l}\text { Concen- } \\
\text { tration } \\
\text { (at. \%) }\end{array}$ & $\begin{array}{c}\text { Peak } \\
\text { Assignment }\end{array}$ \\
\hline$\ldots \mathrm{a}$ & $\mathrm{Au} 4 f_{7 / 2}$ & 83.92 & 0.98 & 2200 & 10.67 & $\ldots$ & $\ldots$ \\
\hline$\ldots \mathrm{b}$ & $\mathrm{Au} 4 f_{7 / 2}$ & 83.92 & 1.6 & 6000 & 10.67 & $\cdots$ & $\cdots$ \\
\hline$\ldots c$ & $\mathrm{Cu} 3 s$ & 122.36 & 3.0 & 1600 & 1.05 & $\ldots$ & $\ldots$ \\
\hline$\ldots \mathrm{b}$ & $\mathrm{Cu} 2 p_{3 / 2}$ & 932.45 & 1.78 & 4000 & 9.73 & $\ldots$ & $\ldots$ \\
\hline
\end{tabular}

a Spot size $300 \mu \mathrm{m}$, pass energy $50 \mathrm{eV}, 2$ scans

${ }^{\mathrm{b}}$ Spot size $800 \mu \mathrm{m}$, pass energy $150 \mathrm{eV}, 1$ scan

${ }^{c}$ Spot size $800 \mu \mathrm{m}$, pass energy $150 \mathrm{eV}, 3$ scans

\section{GUIDE TO FIGURES}

\begin{tabular}{|c|c|c|c|c|c|}
\hline $\begin{array}{l}\text { Spectrum } \\
\text { (Accession) \# }\end{array}$ & $\begin{array}{l}\text { Spectral } \\
\text { Region }\end{array}$ & $\begin{array}{l}\text { Voltage } \\
\text { Shift* }^{\star}\end{array}$ & Multiplier & Baseline & Comment \# \\
\hline $767-1$ & Survey & 0 & 1 & 0 & \\
\hline $767-2$ & Si $2 p$ & 0 & 1 & 0 & \\
\hline $767-3$ & $\mathrm{C} 1 s$ & 0 & 1 & 0 & \\
\hline 767-4 & $\mathrm{O} 1 s$ & 0 & 1 & 0 & \\
\hline $767-5$ & $\mathrm{~F} 1 s$ & 0 & 1 & 0 & \\
\hline
\end{tabular}

* Voltage shift of the archived (as-measured) spectrum relative to the printed figure. The figure reflects the recommended energy scale correction due to a calibration correction, sample charging, flood gun, or other phenomenon. 


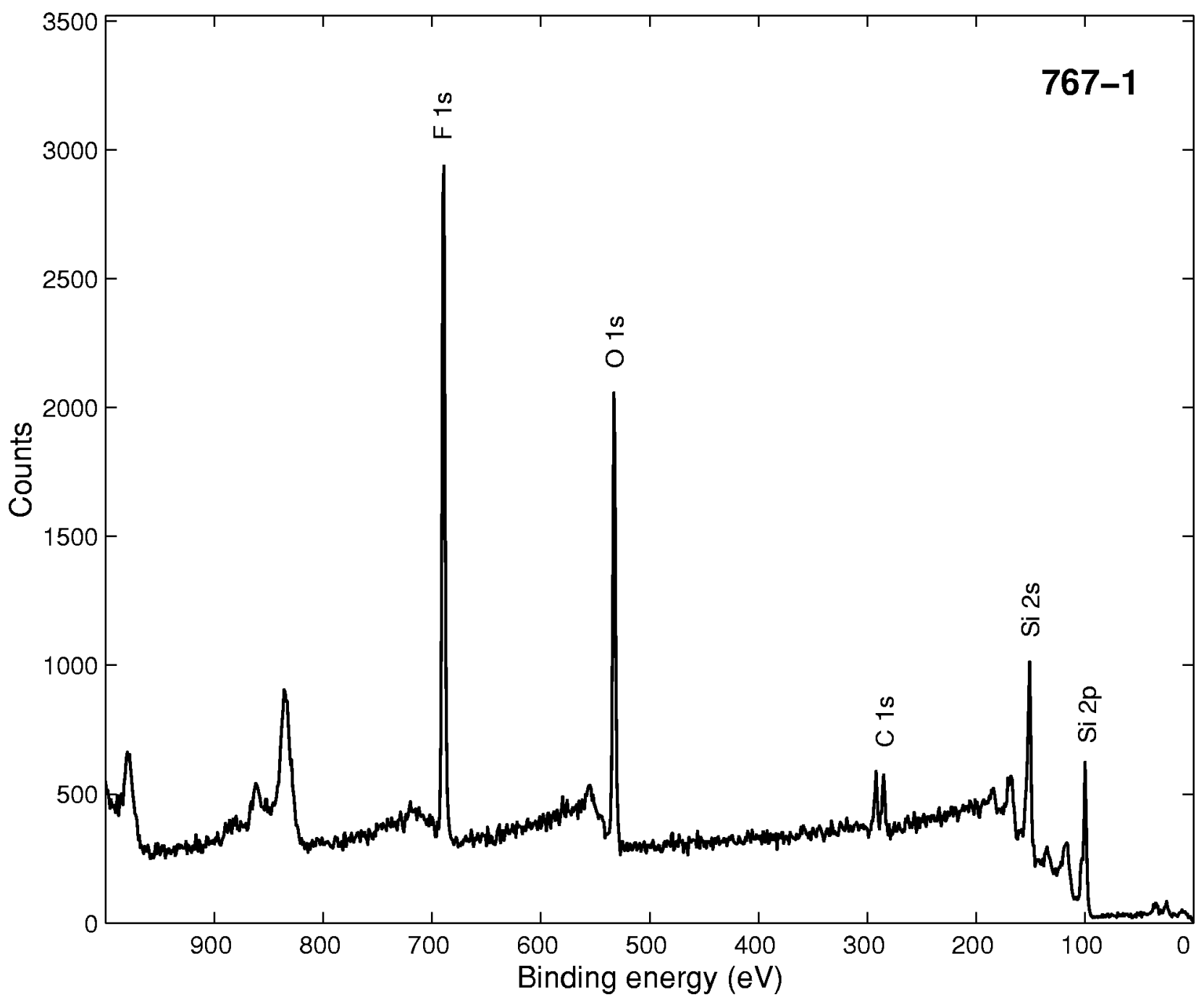

\begin{tabular}{|c|c|}
\hline Accession \# & $00767-01$ \\
\hline Host Material & Monolayer (Tridecafluoro-1,1,2,2-tetrahydrooctyl)-1 dimethylchlorosilane/Si \\
\hline Technique & XPS \\
\hline Spectral Region & survey \\
\hline Instrument & Surface Science Laboratories, Inc. SSX-100 \\
\hline Excitation Source & $\mathrm{Al} K_{\alpha}$ monochromatic \\
\hline Source Energy & $1486.6 \mathrm{eV}$ \\
\hline Source Strength & $200 \mathrm{~W}$ \\
\hline Source Size & $0.8 \mathrm{~mm} \times 0.8 \mathrm{~mm}$ \\
\hline Analyzer Type & spherical sector \\
\hline Incident Angle & $55^{\circ}$ \\
\hline Emission Angle & $55^{\circ}$ \\
\hline Analyzer Pass Energy & $150 \mathrm{eV}$ \\
\hline Analyzer Resolution & $1.5 \mathrm{eV}$ \\
\hline Total Signal Accumulation Time & $1320 \mathrm{~s}$ \\
\hline Total Elapsed Time & $1520 \mathrm{~s}$ \\
\hline Number of Scans & 6 \\
\hline Effective Detector Width & $15.1 \mathrm{eV}$ \\
\hline Analyzer Width & $1500 \mu \mathrm{m} \times 12000 \mu \mathrm{m}$ \\
\hline Comment & note $\mathrm{C} 1 s$ split peak and a strong $\mathrm{F} 1 s$ peak \\
\hline
\end{tabular}



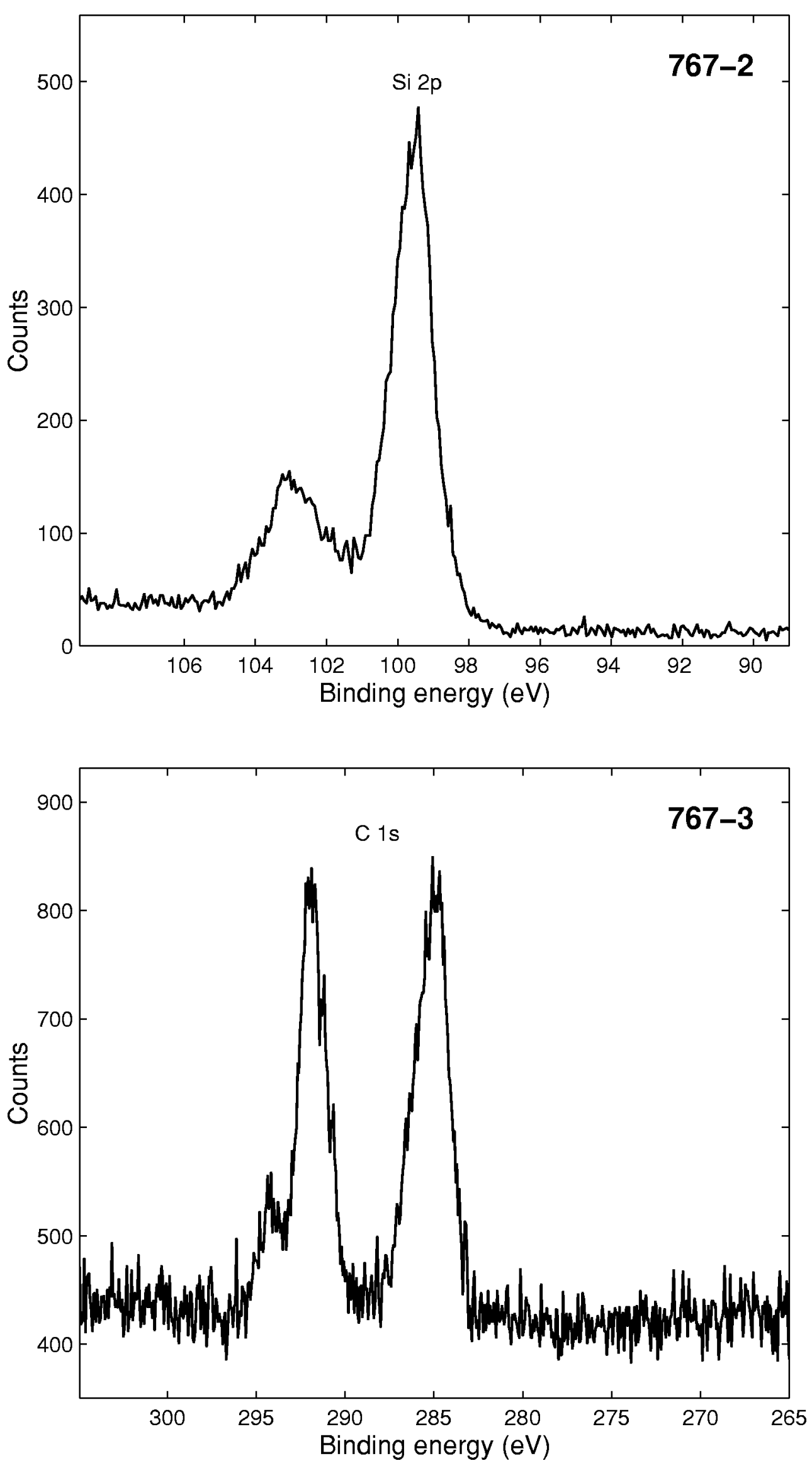

- Accession \#: 00767-02

- Host Material: Monolayer (Tridecafluoro-1,1,2,2tetrahydrooctyl)-1 dimethylchlorosilane/Si

- Technique: XPS

- Spectral Region: Si $2 p$ Instrument: Surface Science Laboratories, Inc. SSX-100

Excitation Source: Al $K_{\alpha}$ monochromatic

Source Energy: $1486.6 \mathrm{eV}$

Source Strength: $200 \mathrm{~W}$

Source Size: $0.8 \mathrm{~mm} \times 0.8 \mathrm{~mm}$ Incident Angle: $55^{\circ}$

Analyzer Type: spherical sector Analyzer Pass Energy: $50 \mathrm{eV}$ Analyzer Resolution: $0.5 \mathrm{eV}$

Emission Angle: $55^{\circ}$

Total Signal Accumulation Time: $368 \mathrm{~s}$

Total Elapsed Time: $537 \mathrm{~s}$

Number of Scans: 6

Effective Detector Width: $6.09 \mathrm{eV}$ Analyzer Width: $750 \mu \mathrm{m} \times$ $6000 \mu \mathrm{m}$

- Accession \#: 00767-03

- Host Material: Monolayer (Tridecafluoro-1,1,2,2tetrahydrooctyl)-1 dimethylchlorosilane/Si

- Technique: XPS

- Spectral Region: C $1 \mathrm{~s}$ Instrument: Surface Science Laboratories, Inc. SSX-100

Excitation Source: $\mathrm{Al} K_{\alpha}$ monochromatic

Source Energy: $1486.6 \mathrm{eV}$

Source Strength: $200 \mathrm{~W}$

Source Size: $0.8 \mathrm{~mm} \times 0.8 \mathrm{~mm}$ Incident Angle: $55^{\circ}$

Analyzer Type: spherical sector Analyzer Pass Energy: $50 \mathrm{eV}$

Analyzer Resolution: $0.5 \mathrm{eV}$

Emission Angle: $55^{\circ}$

Total Signal Accumulation Time: $1227 \mathrm{~s}$

Total Elapsed Time: $1396 \mathrm{~s}$

Number of Scans: 20

Effective Detector Width: $6.09 \mathrm{eV}$

Analyzer Width: $750 \mu \mathrm{m} \times 6000$ $\mu \mathrm{m}$

Comment: Three carbon species are visible: $-\mathrm{CH}_{2},-\mathrm{CF}_{2},-\mathrm{CF}_{3}$ 


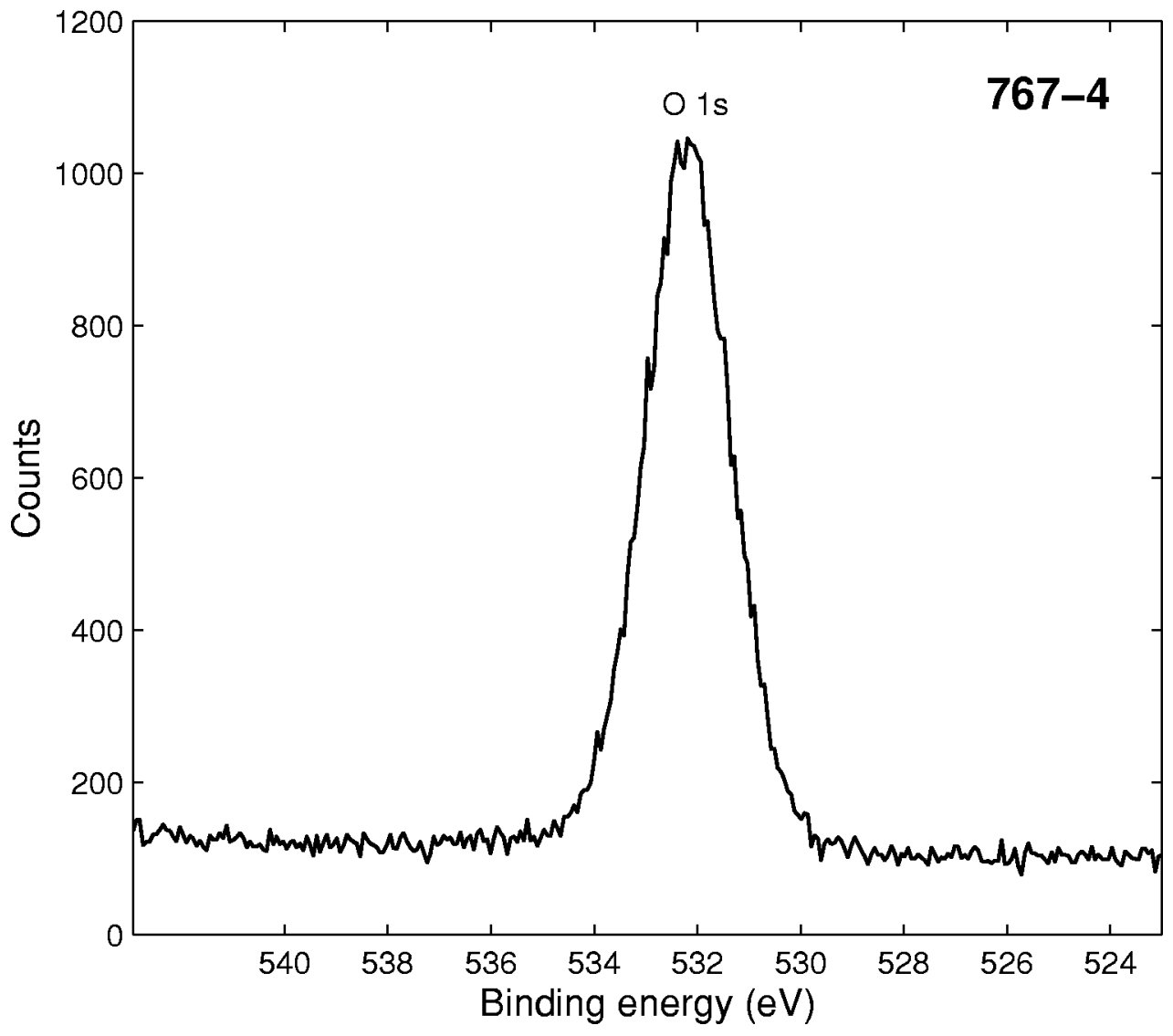

- Accession \#: $00767-04$

- Host Material: Monolayer (Tridecafluoro-1,1,2,2tetrahydrooctyl)-1 dimethylchlorosilane/Si

- Technique: XPS

- Spectral Region: $01 \mathrm{~s}$ Instrument: Surface Science Laboratories, Inc. SSX-100

Excitation Source: Al $K_{\alpha}$ monochromatic

Source Energy: $1486.6 \mathrm{eV}$

Source Strength: $200 \mathrm{~W}$

Source Size: $0.8 \mathrm{~mm} \times 0.8 \mathrm{~mm}$ Incident Angle: $55^{\circ}$

Analyzer Type: spherical sector Analyzer Pass Energy: $50 \mathrm{eV}$

Analyzer Resolution: $0.5 \mathrm{eV}$

Emission Angle: $55^{\circ}$

Total Signal Accumulation Time: $368 \mathrm{~s}$

Total Elapsed Time: $537 \mathrm{~s}$

Number of Scans: 6

Effective Detector Width: $6.09 \mathrm{eV}$

Analyzer Width: $750 \mu \mathrm{m} \times 6000$ $\mu \mathrm{m}$

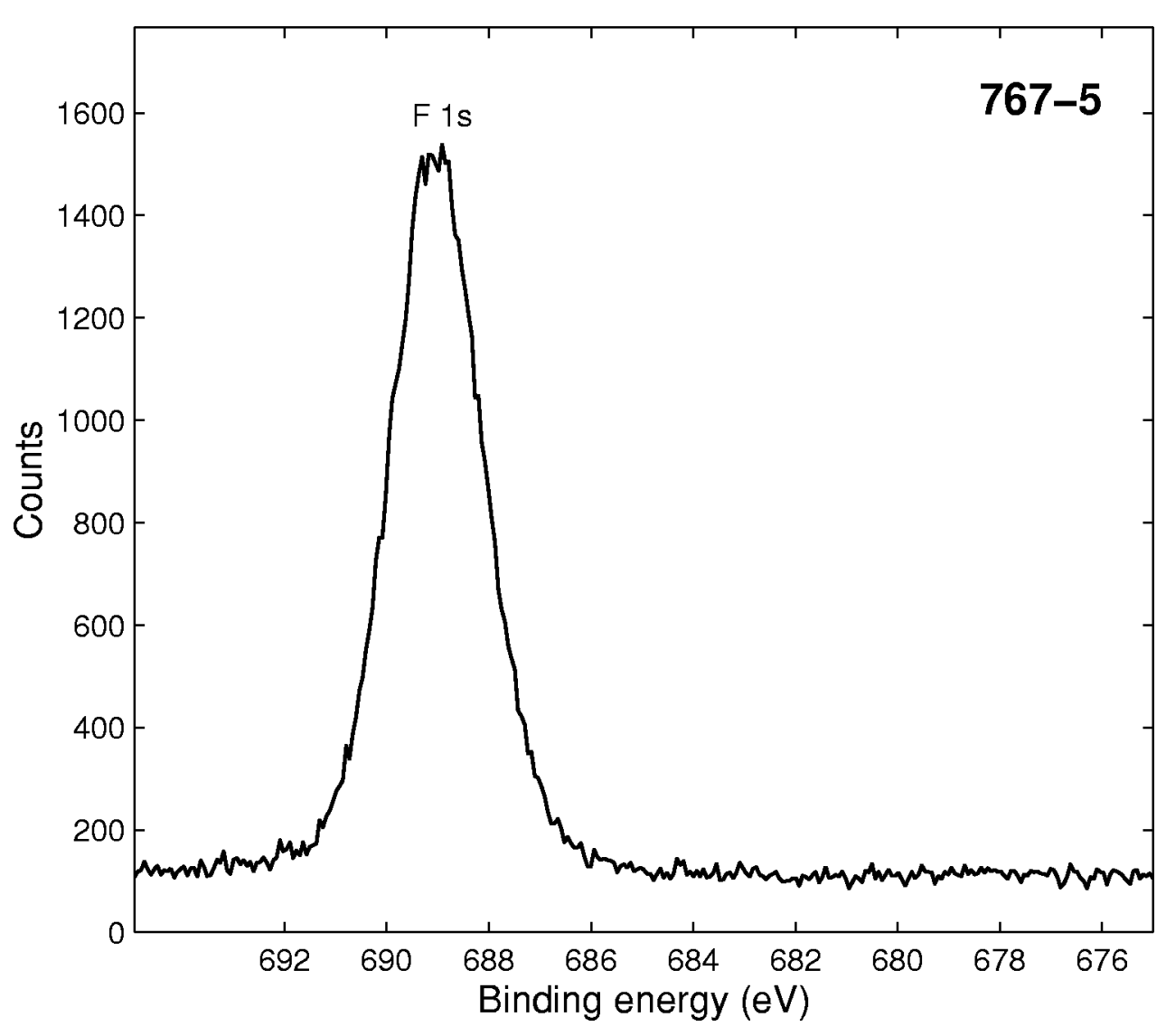

- Accession \#: 00767-05

- Host Material: Monolayer (Tridecafluoro-1,1,2,2tetrahydrooctyl)-1 dimethylchlorosilane/Si

- Technique: XPS

- Spectral Region: F $1 \mathrm{~s}$ Instrument: Surface Science Laboratories, Inc. SSX-100

Excitation Source: Al $K_{\alpha}$ monochromatic

Source Energy: $1486.6 \mathrm{eV}$

Source Strength: $200 \mathrm{~W}$

Source Size: $0.8 \mathrm{~mm} \times 0.8 \mathrm{~mm}$ Incident Angle: $55^{\circ}$

Analyzer Type: spherical sector Analyzer Pass Energy: $50 \mathrm{eV}$ Analyzer Resolution: $0.5 \mathrm{eV}$

Emission Angle: $55^{\circ}$

Total Signal Accumulation Time: $368 \mathrm{~s}$

Total Elapsed Time: $537 \mathrm{~s}$

Number of Scans: 6

Effective Detector Width: $6.09 \mathrm{eV}$ Analyzer Width: $750 \mu \mathrm{m} \times 6000$ $\mu \mathrm{m}$ 